\title{
On Certain Compact Submanifolds of Euclidean Space
}

\author{
Kohei Hatsuse
}

Let $M^{n}$ be a differentiable manifold. When $f$ is an immersion of $M^{n}$ into a Euclidean space $E^{m}$, we can define a function $L_{p}$ on $M^{n}$ by

$$
L_{p}(x)=<p-f(x), p-f(x)>\quad x \in M^{n}
$$

where $<,>$ denotes the Euclidean inner product in $E^{m}$. For almost all points $p \in E^{m}, L_{p}$ is a Morse function on $M^{n}$. Nomizu and Rodriguez [2] proved the following

THEOREM. Let $M^{n}, n \geqq 2$, be a compact connected manifold immersed into $E^{m}$. If every Morse function $L_{p}$ on $M^{n}$ has exactly two critical points, then $M^{n}$ is imbedded as a Euclidean $n$-sphere $S^{n}$.

If a Morse function $L_{p}$ on $M^{n}$ has exactly two critical points, then both of the numbers $C_{0}\left(L_{p}\right)$ of critical points of index zero and $C_{n}\left(L_{p}\right)$ of critical points of index $n$ are equal to one. The purpose of this note is to investigate $M^{n}$ such that $C_{0}\left(L_{p}\right)$ $=1$ or $C_{n}\left(L_{p}\right)=1$ for every Morse function $L_{p}$ on $M^{n}$.

We shall mean $C^{\infty}$ differentiable by "differentiable" and an $n(\geqq 2)$-dimensional differentiable manifold $M^{n}$ by a manifold $M^{n}$.

\section{Lemmas}

The notions and notations will follow from Milnor [1]. Let $f: M^{n} \rightarrow E^{m}$ be an immersion and let $\perp(M)$ be the normal bundle to $M^{\prime \prime}$. If $L_{p}$ is a Morse function on $M^{n}$, then $p \in E^{m}$ is not a focal point of $M^{n}$. Let $x \in M^{n}$ and let $e(x) \in \perp(M)$, that is, a vector normal to $f\left(M^{n}\right)$ at $f(x)$. We denote by $A_{e(x)}$ a symmetric linear transformation of a tangent space $T_{x} M^{n}$ into itself which corresponds to the second fundamental form of $M^{n}$. If $x$ is a critical point of Morse function $L_{p}$ on $M^{n}$ and if $p=f(x)+t e(x), t>0$, then the index at $x$ is equal to the number of eigenvalues $\alpha$ of $A_{e(x)}$ such that $0<1 / \alpha<t$, counting multiplicities.

We shall identify $x \in M^{n}$ with $f(x)$ if there is no confusion. We begin with the following

LemMA 1. Let $M^{n}$ be a manifold immersed into $E^{n+1}$. Then $M^{n}$ is convex if and only if there exists a unit vector $e(x)$ normal to $M^{n}$ at each $x \in M^{n}$ and

Received January 20, 1977.

* Department of Mathematcs, Faculty of Science, Ibaraki University, Mito, Japan. 


$$
L_{p(t, x)}(x) \leqq L_{p(t, x)}\left(y^{\prime}\right) \quad y \in M^{n}
$$

for all $t>0$ where $p(t, x)=x+t e(x)$.

Proof. Let $x \in M^{n}$. If $M^{n}$ is convex then there exists a unit vector $e(x)$ normal to $M^{\prime \prime}$ at $x$ such that $\left\langle e(x), y-x>\leqq 0\right.$ for all $y \in M^{n}$. Let $t>0$ then

$$
L_{p(t, x)}(y)-L_{p(t, x)}(x)=2 t<e(x), x-y>+<x-y, x-y>\geqq 0 .
$$

Therefore $L_{p(t, x)}(x) \leqq L_{p(t, x)}(y)$. Conversely, we suppose that there exists a unit vector $e(x)$ normal to $M^{n}$ and $L_{p(t, x)}(x) \leqq L_{p(t, x)}(y)$ for all $t>0$. Then $<e(x)$, $y-x>\leqq 0$ for all $y \in M^{n}$. Because, if there exists a point $y_{0} \in M^{n}$ such that $<e(x), y_{0}-x>>0$, then

$$
L_{p(t, x)}(x)>L_{p(t, x)}\left(y_{0}\right)
$$

for $t><x-y_{0}, x-y_{0}>/ 2<e(x), y_{0}-x>$. Therefore $M^{n}$ is convex.

Lemma 2. Let $M^{n}$ be a convex manifold immersed into $E^{n+1}$. Let $e(x)$ be a unit vector normal to $M^{n}$ at $x$ as in Lemma 1 . Then every eigenvalue of $A_{e(x)}$ is non-positive.

Proof. The mapping $e: M^{n} \ni x \rightarrow e(x) \in \perp(M)$ defines a differentiable vector field along $M^{n}$. We suppose that $A_{e(x)}$ has a positive eigenvalue. Let $\alpha$ be the largest positive eigenvalue of $A_{c(x)}$ whose multiplicity is $k$, and let $\beta$ be the next largest positive eigenvalue of $A_{e(x)}$. Take $t>0$ such that $1 / \alpha<t<1 / \beta$ (if $\alpha$ is the only positive eigenvalue, just considered $1 / \alpha<t$ ). Then, from Lemma of [2], there exists a point $x^{\prime} \in M^{\prime \prime}$ and it is a critical point of a Morse function $L_{p\left(t, x^{\prime}\right)}$ on $M^{n}$ of index $k$. On the other hand, Lemma 1 implies the index at $x^{\prime}$ is zero. Therefore Lemma is proved.

Lemma 3. Let $M^{n}$ be a compact connected manifold immersed into $E^{n+1}$. If $M^{n}$ is convex then $M^{n}$ is diffeomorphic to $n$-sphere $S^{n}$.

Proof. Since $M^{n}$ is compact, there exists an open ball $D$ of radius $r$ about the origin of $E^{n+1}$ such that $M^{n} \subset D$. The boundary of $D$ is an $n$-sphere $S^{n}$. Every ray $p(t, x)=x+t e(x), t>0$, starting from $x \in M^{n}$ meets $S^{n}$ at only one point $p(t(x), x)$. If we define a mapping $\phi: M^{n} \rightarrow S^{n}$ by

$$
\phi(x)=p(t(x), x) \quad x \in M^{n}
$$

then $\phi$ is differentiable since

$$
t(x)=-\left\langle e(x), x>+\left\{r^{2}-<x, x>+<e(x), x>^{2}\right\}^{1 / 2} .\right.
$$

We denote by exp the exponential mapping of $\perp(M)$ into $E^{n+1}$ and denote by $\pi$ the projection of $\perp(M)$ into $M^{n}$. Then, by the definition of $\phi$, we obtain $\phi(x)=\exp t(x) e(x)$. Let $\left(v^{1}, \ldots, v^{n+1}, U\right)$ be a cubical coordinate system centered 
at $\phi(x)$ in $E^{n+1}$ such that $U \cap S^{n}$ is an $n$-dimensional slice defined by $v^{n+1}$ $=v^{n+1}(\phi(x))$. Let $\left(u^{1}, \ldots, u^{n}, V\right)$ be a coordinate system at $x$ in $M^{n}$ such that $\phi(V) \subset U \cap S^{n}$. There exists a function $u$ in $\perp(M)$, and $u^{1} \circ \pi, \ldots, u^{n} \circ \pi, u$ form a system of coordinates at $t(x) e(x)$. By virtue of Lemma 2, exp is regular at $t(x) e(x)$. Therefore, $\phi$ is a univalent mapping of $V$ into $U \cap S^{n}$, and $u^{i} \circ \phi^{-1}=u^{i} \circ \pi \circ \exp ^{-1}$ | $\phi(V)$ is a differentiable function of $v^{1}, \ldots, v^{n}$. This implies $\phi$ is an immersion. Consequently, $\phi$ is a diffeomorphism of $M^{n}$ into $S^{n}$ since $S^{n}$ is simply connected when $n \geqq 2$.

Lemma of Nomizu and Rodriguez [2] can be stated as in the following

Lemma ([2], p. 119). Let $M^{n}$ be a manifold immersed into $E^{m}$. Let $p \in E^{m}$ and assume that $L_{p}$ has a non-degenerate critical point $x \in M^{n}$ of index $k$. Then there exist sequences $\left\{x_{\lambda}\right\}$ of points in $M^{n}$ and $\left\{p_{;}\right\}$of points in $E^{m}$ such that

(1) $\left\{x_{\lambda}\right\}$ and $\left\{p_{\lambda}\right\}$ converge to $x$ and $p$ respectively,

(2) $L_{p \lambda}$ is a Morse function on $M^{n}$, and $x_{\lambda}$ is a critical point of $L_{p \lambda}$ of index $k$.

\section{Theorems}

Lemma 4. Let $M^{n}$ be a manifold immersed into $E^{m}, m>n+1$. Let $x \in M^{\prime \prime}$ and let $e(x)$ be a unit vector normal to $M^{n}$ at $x$. We put $p(t, x)=x+t e(x)$ when $t>0$ and $q(s, x)=x+s e(x)$ when $s<0$. Then $M^{n}$ belongs to a linear variety $E^{m-1}$ if one of the following properties holds for every unit normal vector $e(x)$ at $x$ :

(a)

$$
L_{p(t, x)}(x) \leqq L_{p(t, x)}(y) \quad y^{\prime} \in M^{n}
$$

for all $t>0$.

$$
L_{q(s, x)}(x) \leqq L_{q(s, x)}(y) \quad y \in M^{n}
$$

for all $s<0$.

Proof. Let $S$ be a set of all unit vectors $e(x)$ normal to $M^{n}$ at $x$. Then $S$ can be considered as a Euclidean $(m-n-1)$-sphere. We suppose that there exists a unit vector $e^{\prime}(x)$ normal to $M^{n}$ at $x$ such that (a) does not hold for it. If we denote by $B$ a set of such unit vectors $e^{\prime}(x)$, then $B$ is an open subset of $S$. Because, if $B$ is not an open subset of $S$, there exists a sequence $\left\{e_{\lambda}(x)\right\}$ of unit vectors in $S-B$ which converges to a suitable $e^{\prime}(x) \in B$. For each $e_{\lambda}(x)$, (a) holds. Therefore

$$
\begin{aligned}
L_{p^{\prime}(t, x)}(x) & =\lim _{\lambda} L_{p_{\lambda}(t, x)}(x) \\
& \leqq \lim _{\lambda} L_{p_{2}(t, x)}(y)=L_{p^{\prime}(t, x)}(y)
\end{aligned}
$$


for all $t>0$ where $p^{\prime}(t, x)=x+t e^{\prime}(x)$ and $p_{\lambda}(t, x)=x+t e_{\lambda}(x)$. This contradicts $e^{\prime}(x) \in B$.

When $e^{\prime}(x) \in B$, (a) holds for $-e^{\prime}(x)$ and hence $B \neq S$. Let $e(x)$ be a boundary point of $B$. Then there exists a sequence $\left\{e_{\mu}^{\prime}(x)\right\}$ of unit vectors in $B$ which converges to $e(x)$. For each $e_{\mu}^{\prime}(x)$, (b) holds. Therefore

$$
\begin{aligned}
L_{q(s, x)}(x) & =\lim _{\mu} L_{q_{\mu}^{\prime}(s, x)}(x) \\
& \leqq \lim _{\mu} L_{q_{\mu}^{\prime}(s, x)}(y)=L_{q(s, x)}(y)
\end{aligned}
$$

for all $s<0$ where $q_{\mu}^{\prime}(s, x)=x+s e_{\mu}^{\prime}(x)$. Since $B \neq S$, we obtain $e(x) \notin B$. These imply both of (a) and (b) hold for $e(x)$. Therefore

$$
<e(x), y-x>=0
$$

for all $y \in M^{n}$. Thus $M^{n}$ belongs to a linear variety $E^{m-1}$ perpendicular to $e(x)$.

THEOREM 1. Let $M^{n}$ be a compact connected manifold immersed into $E^{m}$. Let $e(x) \in \perp(M)$ and let $\alpha_{1}, \ldots, \alpha_{n}$ be eigenvalues of $A_{\mathrm{e}(x)}$. Assume that

(1) $\alpha_{i} \alpha_{j} \geqq 0(i, j=1, \ldots, n)$ for every $e(x) \in \perp(M)$,

(2) $C_{0}\left(L_{p}\right)=1$ for every Morse function $L_{p}$ on $M^{n}$.

Then $M^{n}$ is diffeomorphic to $n$-sphere $S^{n}$.

Proof. Let $e(x) \in \perp(M)$ be a unit vector at $x \in M^{n}$. We suppose that eigenvalues $\alpha_{1}, \ldots, \alpha_{n}$ of $A_{e(x)}$ are non-positive. Let $t>0$. Then $x$ is a non-degenerate critical point of $L_{p(t, x)}$ of index zero. There exist sequences $\left\{x_{\lambda}\right\}$ of points in $M^{n}$ and $\left\{p_{\lambda}\right\}$ of points in $E^{m}$ as in Lemma of [2]. Since $C_{0}\left(L_{p_{\lambda}}\right)=1$ from (2), Morse function $L_{p_{\lambda}}$ attains to minimal value at exactly one point $x_{\lambda}$. Therefore

$$
L_{p(t, x)}(x)=\lim _{\lambda} L_{p_{\lambda}}\left(x_{\lambda}\right) \leqq \lim _{\lambda} L_{p_{\lambda}}(y)=L_{p(t, x)}(y)
$$

for all $t>0$. From (1), this implies one of the properties (a) and (b) in Lemma 4 holds for every unit vector $e(x)$ normal to $M^{n}$. According to Lemma $4, M^{n}$ belongs to a linear variety $E^{n+1}$. When $M^{n} \subset E^{n+1}$, the properties (a) and (b) for every unit vector $e(x) \in \perp(M)$ imply $M^{n}$ is immersed into $E^{n+1}$ as a convex hypersurface from Lemma 1. Thus, from Lemma $3, M^{n}$ is diffeomorphic to $n$-sphere $S^{n}$.

THEOREM 2. Let $M^{n}$ be a compact connected manifold immersed into $E^{m}$. Let $e(x) \in \perp(M)$ and let $\alpha_{1}, \ldots, \alpha_{n}$ be eigenvalues of $A_{e(x)}$. We denote by $N$ a set of $e(x) \in \perp(M)$ such that $\alpha_{i} \alpha_{j}>0(i, j=1, \ldots, n)$. Assume that

(1) $\perp(M)=\bar{N}$,

(2) $C_{n}\left(L_{p}\right)=1$ for every Morse function $L_{p}$ on $M^{n}$. 
Then $M^{n}$ is diffeomorphic to $n$-sphere $S^{n}$.

Proof. Let $e(x) \in \perp(M)$ be a unit vector at $x \in M^{n}$. We suppose that eigenvalues $\alpha_{1}, \ldots, \alpha_{n}$ of $A_{e(x)}$ are positive. Let $\alpha_{1}$ be the smallest eigenvalue of $A_{e(x)}$. If $t>1 / \alpha_{1}$ then $x$ is a non-degenerate critical point of $L_{p(t, x)}$ of index $n$. There exist sequences $\left\{x_{\lambda}\right\}$ of points in $M^{n}$ and $\left\{p_{\lambda}\right\}$ of points in $E^{n}$ as in Lemma of [2]. Since $C_{n}\left(L_{p_{\lambda}}\right)=1$ from (2), Morse function $L_{p_{\lambda}}$ attains to maximal value at exactly one point $x_{\lambda}$. Therefore

$$
L_{p(t, x)}(x)=\lim _{\lambda} L_{p_{\lambda}}\left(x_{\lambda}\right) \geqq \lim _{\lambda} L_{p_{\lambda}}(y)=L_{p(t, x)}(y)
$$

and hence $<e(x), x-y>\leqq 0$ for all $y \in M^{n}$. If $s<0$ then we obtain

$$
L_{q(s, x)}(x)-L_{q(s, x)}(y)=-2 s<e(x), x-y>-<x-y, x-y>\leqq 0
$$

for all $y \in M^{n}$. By the definition of $N$, this implies one of the properties (a) and (b) in Lemma 4 holds for every unit vector $e(x) \in N$.

When $e(z) \in \perp(M)$ is a unit vector at $z \in M^{n}$, there exists a sequence $\left\{e_{\mu}\left(x_{\mu}\right)\right\}$ of unit vectors in $N$ which converges to $e(z)$. If (a) holds for all $e_{\mu}\left(x_{\mu}\right)$, then

$$
\begin{aligned}
L_{p(t, z)}(z) & =\lim _{\mu} L_{p_{\mu}(t, x \mu)}\left(x_{\mu}\right) \\
& \leqq \lim _{\mu} L_{p_{\mu}(t, x \mu)}(y)=L_{p(t, z)}(y)
\end{aligned}
$$

for all $t>0$ and $y \in M^{n}$ where $p_{\mu}\left(t, x_{\mu}\right)=x_{\mu}+t e_{\mu}\left(x_{\mu}\right)$. Similarly, if (b) holds for all $e_{\mu}\left(x_{\mu}\right)$ then (b) holds for $e(z)$. Thus one of the properties (a) and (b) in Lemma 4 holds for every unit vector $e(x)$ normal to $M^{n}$. According to Lemmas 1 and $4, M^{n}$ belongs to a linear variety $E^{n+1}$, and it is immersed into $E^{n+1}$ as a convex hypersurface. Consequently, from Lemma $3, M^{n}$ is diffeomorphic to $n$-sphere $S^{n}$.

\section{References}

[1] J. Milnor, Morse theory, Ann. of Math. Studies, No. 51, Princeton University Press, 1963.

[2] K. Nomizu and L. Rodrgíuez, Umbilical submanifolds and Morse functions, Nagoya Math. J., 48 (1972), 197-201. 